\title{
Lumen
}

Selected Proceedings from the Canadian Society for Eighteenth-Century Studies

\section{Les notes du traducteur des Voyages de Gulliver : détonation et " détonnement "}

\section{Benoit Léger}

Volume 21, 2002

URI : https://id.erudit.org/iderudit/1012274ar

DOI : https://doi.org/10.7202/1012274ar

Aller au sommaire du numéro

Éditeur(s)

Canadian Society for Eighteenth-Century Studies / Société canadienne d'étude du dix-huitième siècle

ISSN

1209-3696 (imprimé)

1927-8284 (numérique)

Découvrir la revue

Citer cet article

Léger, B. (2002). Les notes du traducteur des Voyages de Gulliver : détonation et " détonnement ". Lumen, 21, 179-198. https://doi.org/10.7202/1012274ar

Copyright (c) Canadian Society for Eighteenth-Century Studies / Sociéte canadienne d'étude du dix-huitième siècle, 2002
Ce document est protégé par la loi sur le droit d'auteur. L'utilisation des services d'Érudit (y compris la reproduction) est assujettie à sa politique d'utilisation que vous pouvez consulter en ligne.

https://apropos.erudit.org/fr/usagers/politique-dutilisation/ 


\section{Les notes du traducteur des Voyages de Gulliver: détonation et «détonnement»}

Cet article ${ }^{1}$ aborde le rôle d'un élément peu étudié du paratexte des traductions au XVIII ${ }^{e}$ siècle, soit le corpus des notes du traducteur, à partir du cas de la traduction des Voyages de Gulliver par Pierre-François Guyot, abbé Desfontaines (1685-1745), publiée d'abord en $1727^{2}$. Cette traduction a fait l'objet de nombreuses critiques, en fait le plus souvent des comparaisons minutieuses de l'original et de la traduction ${ }^{3}$ qui montrent les «infidélités» de la traduction. Ces critiques relèvent en fait des jugements traditionnels sur la traduction, jugements parfois «engagés ${ }^{4} »$, teintés de platonisme, et où l'idée d'un original, génial et intouchable, est plus ou moins latente, de même que celle d'une conception souvent ambiguë de la «fidélité» en traduction.

Notre lecture de la traduction de Desfontaines tient compte de ce que Berman définit comme l'«analyse descriptive» et comme la "critique des traduction $s^{5} »$. De l'analyse descriptive, nous reprenons le souci de re-

1 Les recherches qui ont mené au présent article ont été rendues possibles grâce à une subvention du Fonds pour la formation de chercheurs et l'aide à la recherche du gouvernement du Québec. L'auteur tient à remercier son assistante de recherche, Janine Hopkinson.

2 Jonathan Swift, Voyages de Gulliver, Paris, Jacques Guérin, 1727. Sauf mention contraire, l'édition utilisée ici est celle de Garnier dans les Voyages imaginaires, Paris, 1787, qui reprend la plupart des notes de Desfontaines, ainsi que la préface du traducteur de l'édition revue et corrigée de 1727. L'orthographe des citations n'a pas été modifiée.

3 Voir Thelma Morris, "L'abbé Desfontaines et son rôle dans la littérature de son temps", Studies on Voltaire and the Eighteenth Century, 19, 1961; Sybil Goulding, Swift en France, Paris, Champion, 1924.

4 Antoine Berman, Pour une critique des traductions: John Donne, Paris, Gallimard, 1995, 45.

5 Ibid., 38-44, 50-63. 
mettre en contexte une traduction produite à une époque où la censure influe sur la publication des textes et où l'obsession du "goût du public» oriente la manière de traduire de Desfontaines (du moins dans son discours officiel), tout comme son propre souci du succès commercial. Ce respect du contexte de production de la traduction implique que l'on souligne que la traduction de Gulliver's Travels est non seulement la première en France, mais l'une des premières publications en France $\mathrm{d}^{\prime}$ un texte clairement attribué à Swift ${ }^{6}$ - la traduction de La Haye évoquée plus loin connaîtra une réception et une diffusion très limitées en France ${ }^{7}$. En cela, le texte de Desfontaines constitue une "traductionintroduction ${ }^{8}$. De la critique des traductions, nous reprenons ce que Berman décrit comme la deuxième étape, soit la définition de la «position traductive du traducteur ${ }^{9} »$, du «projet de traduction ${ }^{10} »$ et de «l'horizon du traducteur ${ }^{11} \gg$.

Après la mise au point des circonstances de publication de la traduction, nous montrerons, dans un deuxième temps, comment les notes du traducteur dans les Voyages de Gulliver entrent en contradiction avec la position traductive officielle de Desfontaines telle qu'orientée par son horizon de traduction et affichée dans sa préface.

\section{Contexte de la traduction}

Pour comprendre le fonctionnement du paratexte de la traduction de Desfontaines, il faut d'abord prendre connaissance du contexte de sa

6 Ainsi que l'annonce l'incipit de la préface du traducteur: «L'Auteur de cet Ouvrage est le célébre M. Swift, Irlandois.» La nationalité de Swift sera corrigée dès la deuxième édition. Sur les traductions antérieures (surtout aux Pays-Bas) de textes de Swift, voir Goulding, Swift en France, 1-11.

7 Voir infra.

8 Berman, op. cit., 84 .

9 «[Le] "compromis" entre la manière dont le traducteur perçoit en tant que sujet pris par la pulsion de traduire, la tâche de la traduction, et la manière dont il a "internalisé" le discours ambiant sur le traduire (les "normes")» (ibid., 74).

10 «Le projet définit la manière dont, d'une part, le traducteur va accomplir la translation littéraire, d'autre part, assumer la traduction même, choisir un "mode" de traduction, une "manière de traduire"» (ibid., 76).

11 «D'une part, désignant ce-à-partir-de-quoi l'agir du traducteur a sens et peut se déployer, elle pointe l'espace ouvert de cet agir. Mais, d'autre part, elle désigne ce qui clôt, ce qui enferme le traducteur dans un cercle de possibilités limitées» (ibid., 80). 
parution en avril 1727, moins de six mois après la publication des Travels en Angleterre en octobre 1726. On sait que Voltaire, qui avait rencontré Swift en Angleterre, lit Gulliver's Travels en novembre 1726 et en envoie rapidement un exemplaire à son ami Thiérot, lui suggérant de procéder à la traduction ${ }^{12}$. En février 1727, Voltaire écrit encore à Thiérot et annonce que «quelqu'un de plus pressé» l'a précédé «en traduisant le premier tome qui est fait pour plaire à toutes les nations ${ }^{13} »$. Le ton neutre et l'absence de précision sur le traducteur laissent ici supposer qu'il ne s'agit pas de la traduction de Desfontaines, mais bien de la toute première version française, celle qui paraît, sans mention de traducteur, à La Haye en janvier $1727^{14}$.

Selon David Williams et Thelma Morris ${ }^{15}$, Thiérot aurait annoncé à Desfontaines qu'il allait traduire le Gulliver, mais celui-ci le devancera, bien qu'il ait entendu parler de la traduction de La Haye, encore à paraître. Le Mercure de France, dans une "Addition aux Nouvelles littéraires» de mars 1727, annonce que «M. l'Abbé D. F.» y travaille depuis trois mois ${ }^{16}$. L'annonce du Journal des savants (avril 1727) ${ }^{17}$ est plus succincte et ne donne que le titre et l'auteur: «[1] e celebre Mr Swift, Doyen de l'Église de Saint Patrice de Dublin en Irlande, qui a déjà donné plusieurs autres Ouvrages au Public, \& entr'autres le fameux Conte $d u$ tonneau.» Le traducteur reste anonyme. En fait, au moment de l'annonce du Journal, la traduction de Desfontaines est déjà en vente: elle paraît pendant la deuxième ou la troisième semaine d'avril, soit deux mois et demi seulement après la réception par Thiérot de la lettre de Voltaire ${ }^{18}$.

12 Voltaire, Correspondance, Institut et musée Voltaire, Genève, 1953, vol. 2, lettre 298, 42.

13 Voltaire, ibid., vol. 2, lettre 300, 43-45.

14 Voyages du Capitaine Lemuel Gulliver en divers pays eloignez [...], La Haye, Gosse et Neaulme, 1727.

15 Voir «Essay sur la poésie épique, traduit de l'anglois de M. de Voltaire, par M. ***», Paris, Chaubert, 1728, réédité dans CEuvres complètes de Voltaire, 3B, Oxford, Voltaire Foundation, éd. critique de David Williams, 1996; Morris, op. cit., 278-294.

16 Mercure de France (1724-1794), Paris, Cavelier et Panckoucke, Genève, Slatkine reprints, 1968, 627-628.

17 Soit au moment où Desfontaines est exclu du Journal (voir Jean Sgard, dir., Dictionnaire des journalistes (1600-1789), Grenoble, Presses universitaires, 1976), vraisemblablement avant la parution de l'article, mais peut-être pas au moment de sa rédaction. Journal des Sçavans, par Andry, Bignon, Belley, Bruhier, et al., Paris, $1665-1792,351$.

18 Goulding, op. cit., 60; Morris, op. cit., 279. 


\section{Un livre «agréable \& plein d'esprit»}

L'édition de 1726 des Travels ne comportait qu'une seule instance liminaire: le texte de cinq pages, intitulé «The Publisher to the Reader», signé «Richard Sympson». Il s'agissait de donner des détails sur la façon dont l'éditeur serait entré en contact avec le texte et d'indiquer les modifications et corrections apportées au texte. Dans la version de Desfontaines, la préface de Sympson est remplacée par une dédicace non datée à Mme du Deffand et par sa propre préface du traducteur.

C'est dans cette préface que Desfontaines justifie sa poétique traductionnelle et décrit le résultat escompté ${ }^{19}$. Reprenant plusieurs clichés des préfaces traductionnelles, il raconte la manière dont il serait entré en contact avec le Gulliver, grâce à son ami Francis Atterbury, ainsi que sa première lecture: un «Seigneur Anglois» en aurait reçu un exemplaire à Paris et lui en aurait parlé comme d'un livre «agréable \& plein d'esprit.» D'autres Anglais au jugement sûr auraient ensuite exhorté l'angliciste que devenait Desfontaines à «faire connoître cet ouvrage ingénieux à la France, par une Traduction qui pût répondre à l'Original ${ }^{20}$ ».

La préface pose les éléments d'une poétique officielle dont Desfontaines s'éloignera peu dans de tels textes théoriques. En accord avec la rhétorique préfacielle, il commence par convaincre le lecteur de l'importance de l'auteur, de l'intérêt et de l'originalité de son texte ainsi que de l'utilité de la traduction, pour ensuite souligner les fautes du texte anglais et mieux présenter sa propre solution: la suppression des passages de mauvais goût. Le traducteur cède ensuite la place au critique littéraire pour rejeter, sans l'avoir lue, la traduction de La Haye. La mise en valeur de l'ouvrage à lire dépasse ici la modestie rhétorique habituelle aux préfaces du traducteur et se mue en attaques de type journalistique. La justification du texte de Desfontaines passe donc autant par la valeur intrinsèque du texte de départ que par le travail original d'adaptation par le traducteur (et qu'il faut défendre contre la concurrence). Desfontaines rappelle ensuite ce qui lui a plu dans l'original, s'excuse de ses erreurs et revient sur le naturel des sentiments de Gulliver. Il conclut enfin en demandant pardon au lecteur de n'avoir pas, malgré ses efforts, rendu l'ouvrage assez conforme au goût français.

19 Jonathan Swift, Voyages de Gulliver, trad. Desfontaines, op. cit., xv-xxxii.

20 Desfontaines, Voyages de Gulliver, Paris, Garnier, 1787, xvii. Selon Morris (278), l'Irlandais Markan, qui enseignait alors l'anglais à Desfontaines, faisait partie de ce groupe. 
La préface de Desfontaines n'a d'autre but que d'inscrire la traduction française de Gulliver dans un cadre référentiel compréhensible et acceptable, tant pour le public que pour la critique. Même si c'est la satire qui avait plu à Voltaire, le Gulliver doit ici être présenté comme une fable morale ou, mieux encore, comme une allégorie. L'axe central de cette préface est en fait la résolution de la contradiction inhérente à la position traductive de Desfontaines: il justifie le fait d'avoir traduit le texte par la valeur de celui-ci et par l'importance de l'auteur, mais il doit également rendre compte des modifications qu'il a apportées à ce texte. On pourrait donc penser que le traducteur, soucieux du succès de son ouvrage, adapte ou gomme toutes les allusions satiriques. Mais, nous le verrons dans les notes du traducteur, si la satire est reléguée au second plan et l'allégorie morale mise en valeur dans la préface, Desfontaines ne renonce pourtant pas complètement à la première.

\section{Rôle et fonctions des notes}

La note semble de prime abord un élément du paratexte exempt de signification autonome, dont il est difficile de saisir le rôle. Genette la définit comme un «énoncé de longueur variable [...] relatif à un segment plus ou moins déterminé du texte, et disposé soit en regard soit en référence à ce segment». Elle se distinguerait de la préface, au niveau formel, par le "caractère toujours partiel du texte de référence» et donc par «le caractère local de l'énoncé porté en note ${ }^{21}{ }$. Genette décrit le statut des notes à l'aide du classement qu'il emploie pour les préfaces. Elles peuvent donc être auctoriales assomptives, soit celles de l'auteur qui en assume la responsabilité (cas le plus fréquent); dénégatives ou fictives allographes authentiques (notes de l'éditeur ou du traducteur par exemple); etc.

Si la note ne se distingue guère du texte dans les cas où elle est attribuée à l'auteur, elle pose d'autres problèmes lorsqu'elle est l'œuvre du traducteur; il y a ici de l'éditorial et de l'auctorial réunis dans un même élément du paratexte, peut-être de la manière la plus forte en dehors du texte proprement dit. Ces notes seront classées ici dans la catégorie des notes «auctoriales traductionnelles» (de préférence à «éditoriales» ou "allographes»). Le terme, s'il peut sembler rébarbatif, a l'avantage de bien distinguer la note attribuée au traducteur de celle de 
l'auteur ou de celles qui relèvent de l'édition critique ou savante. Il souligne de plus le caractère original (auctorial) de la traduction.

On peut également adopter la nature du texte principal comme critère et distinguer les notes de textes discursifs de celles de textes de fiction ${ }^{22}$. Les premières définissent ou expliquent des termes, traduisent des citations ou en donnent la source. Or, comme le montre l'exemple de Gulliver's Travels, ce genre de notes n'est pas étranger aux textes de fiction du XVIII ${ }^{\mathrm{e}}$ siècle et la distinction est donc loin d'être claire. La note peut également, en particulier au XVIIIe siècle, receler les éléments les plus polémiques ou les plus sarcastiques du discours.

Les notes de l'auteur (ou du traducteur) d'un texte de fiction ont d'autres fonctions et d'autres effets. Comme le souligne Genette, «l'annotation auctoriale d'un texte de fiction ou de poésie marque inévitablement, par son caractère discursif, une rupture de régime énonciatif...». La note vient en effet détruire la fiction et constitue un "coup de pistolet référentiel dans le concert fictionnel ${ }^{23}{ }^{\prime}$. Il convient de s'interroger sur le rôle d'une telle «détonation» (entendue au sens du coup de feu) et d'un tel «détonnement» (un changement de ton), pour lequel on se permettra ici le néologisme formé à partir de «détonner», soit "sortir du ton», «ne pas être en harmonie (avec un ensemble) ${ }^{24} »$.

Que l'on insiste sur le rôle du traducteur comme critique et donc comme médiateur du texte ou sur son statut d'auteur, de responsable du texte, la détonation et le détonnement influent sur la manière dont le texte sera lu: la détonation, interrompant la lecture, empêche le lecteur de se laisser emporter par les «imaginations» de l'auteur et met l'accent sur le passage; le détonnement, introduisant en apparence une valeur morale, ou une critique des idées avancées par l'auteur, souligne à sa manière l'importance du passage et en force même la relecture ou une lecture plus attentive.

La note du traducteur est donc un phénomène plus complexe qu'il n'y parait de prime abord et qui, jouant un rôle dans l'orientation de la lecture, mérite notre attention. Elle jouit de plus, par son emplacement, d'une liberté plus grande, alors que les dédicaces et les préfaces suivent un modèle rhétorique établi et remplissent des fonctions précises. Selon Suzanne Saïd et Christian Biet, son étude peut même permettre de

22 Ibid., 300 et suiv.

23 Ibid., 305, 307.

24 Voir Alain Rey, dir., Dictionnaire historique de la langue française, Paris, Dictionnaires Le Robert, 1998, vol. 3, 3845, «ton». 
définir «les véritables interlocuteurs d'un traducteur qui cesse pour un moment de s'effacer totalement derrière son auteur ${ }^{25} »$. L'analyse des notes du traducteur peut alors nous faire pénétrer dans ce que les auteurs appellent les «coulisses de la traduction». Dans le cas de textes classiques, comme Antigone, si les lecteurs sont considérés comme savants, la note donne un «en deçà» de la traduction en offrant une version plus littérale, tandis que, s'ils sont considérés comme ignorants, la note va «au-delà» pour développer la traduction par le rappel de "quelques particularités nécessaires à l'intelligence du texte» concernant «l'art et le dessein du poète, les mœurs du pays ou les usages du temps ${ }^{26} »$. Au cours du premier tiers du XVIII ${ }^{e}$ siècle, donc avant l'anglomanie que connaîtra la France, la note du traducteur se contente généralement de «l'au-delà», puisque les lecteurs qui savent l'anglais sont encore une denrée rare.

Le «jeu des notes», dont la fonction ne se limite pas à ce pour quoi elles se donnent, se situe pour Saïd et Biet en fait entre l'esthétique et l'idéologie, car elles forment un «appareil complexe, pédagogique et érudit, qui permet de poser et de résoudre à moindres frais des problèmes esthétiques et idéologiques qui sont d'abord ceux du XVIII siècle ${ }^{27}$ ». Elles sont rendues nécessaires par les «aspérités» qui peuvent surprendre le lecteur et la censure. Le traducteur peut prendre parti pour ou contre le texte et éventuellement censurer l'auteur, mais les jugements esthétiques du traducteur qui critique l'invraisemblance ou le mauvais goût de certains passages (ou qui affirme même les avoir corrigés) peuvent jouer le rôle d'un paravent destiné à faire diversion, à «masquer des problèmes plus aigus et [à] détourner l'attention du lecteur ${ }^{28}{ }$. L'analyse de Saïd et Biet a pour corpus les traductions d'Antigone au XVIII ${ }^{e}$ siècle, «une œuvre capable de troubler un lecteur vivant dans une monarchie par ses attaques furieuses contre le tyran Créon ${ }^{29} »$, mais les textes traduits par Desfontaines eux aussi mettent la société en question, non pas sur le mode tragique, mais sur le mode ironique ou satirique.

Il faut alors se demander comment les notes du traducteur des Travels s'opposent aux mises en garde et aux jugements esthétiques des préfaces; quels sont les passages qui appellent le plus souvent une Sophocle au XVIII 'eiècle», Poétique, 58, 1984, 156.

26 Ibid.

27 Ibid., 165-169, «IV. Le jeu des notes».

28 Ibid., 167

29 Ibid. 
explication ou un commentaire du traducteur; et, surtout, si le «paravent» est vraiment opaque.

Saïd et Biet voient dans les notes du traducteur le premier moyen de «déchiffrer le sens de l'ensemble et [de] repérer le système de lecture et d'interprétation du traducteur ${ }^{30}$ ", ce qui est peut-être possible dans le cas d'un corpus de notes particulièrement volumineux. Dans le cas des Voyages de Gulliver, ce corpus est moins important (ce qui peut être tout aussi significatif), mais le traducteur n'en prend pas moins position. L'hostilité de Desfontaines envers les commentaires qui «grossissent» l'ouvrage fait d'abord partie des enjeux de ses propres notes de traduction. Les allusions à l'érudition stérile sont en effet récurrentes dans les notes et dans le texte proprement dit de ses ouvrages satiriques ou de ses articles. Dans les notes, la satire passe par leur caractère souvent superflu et aléatoire. Le meilleur exemple est celui de la «Lettre du Docteur Ferruginer», annexe au Nouveau Gulliver ${ }^{31}$, où les références gréco-latines plus ou moins fiables s'accumulent ad nauseam. A la même époque, dans un article du Nouvelliste du Parnasse de 1731 consacré au père Brumoy et à son «Discours sur la tragédie ${ }^{32}$ », Desfontaines est encore plus direct: «Ce qui me plait beaucoup, c'est qu'il n'a point noïé le texte dans un abîme de notes, défaut ordinaire à ces petits Scholiastes dont tout le talent est de compiler avec scrupule ${ }^{33}$."

\section{Les notes du Gulliver de Desfontaines}

Précisons d'abord que dans l'édition anglaise de $1726^{34}$, celle utilisée par Desfontaines, les deux derniers Voyages de Gulliver ne comportent aucune note en bas de page, de quelque nature qu'elle soit. Toutes les notes mentionnées plus loin peuvent donc être considérées comme des notes auctoriales traductionnelles. La traduction de Desfontaines, quant à elle, comporte sept notes en bas de page qui se trouvent toutes dans le deuxième tome (voyages à Laputa et chez les Houyhnhnms). Elles

30 Ibid., 169.

31 Desfontaines, le Nouveau Gulliver [...], Paris, Clouzier et Le Breton, 1730.

32 Le Théâtre des Grecs, Paris, Rollin Père, 1730.

33 Le Nouvelliste du Parnasse, ou, Réflexions sur les ouvrages nouveaux (Paris, 1730-1732), Genève, Slatkine reprints, 1967, I, 6, 127-128.

34 Jonathan Swift, Gulliver's Travels, 1726, «Scholars' Facsimiles \& Reprints», introd. de C. McKelvie, New York, Delmar, 1976. 
renvoient donc aux voyages les plus difficiles à saisir pour le traducteur et les lecteurs; ce sont effectivement ceux que les critiques attaqueront particulièrement.

On trouve ici d'abord des notes qui semblent se limiter aux fonctions habituelles de tels éléments paratextuels, telle l'explication de termes anglais «intraduisibles», lorsque Gulliver, dans l'île de Glubbdubdrib, demande aux morts comment ils ont obtenu, de leur vivant, honneurs et richesses. Gulliver rapporte ainsi ses observations: «Parmi ces moyens, je vis le parjure, l'oppression, la subornation, la perfidie, le *Pandarisme \& autres pareilles bagatelles qui méritent peu d'attention.» Le traducteur répond à ce commentaire par une brève note, note censée expliquer le sens d'une expression rare: «*En Anglois Pandarism, mot forgé qu'on rend ici sans le traduire, \& qui s'entend aisément ${ }^{35}$.»

On retrouve également des notes savantes, qui, tout en rapprochant le texte du lecteur, permettent de mettre en évidence l'érudition du traducteur, dont la référence de citations latines, dans la conclusion des Voyages. Lorsque Gulliver s'associe à Sinon («—*Nec si miserum Fortuna Sinonem/Finxit, vanum etiam mendacemque improba finget»), le traducteur ajoute en note la source: «*Virg. Æneïd, I.2. ${ }^{36}$.» Alors que, selon la plupart des critiques anglo-saxons, cette citation (dont la référence n'est pas donnée dans le texte anglais de 1726) a pour fonction de souligner le caractère fictionnel du récit en insistantsur le fait que Gulliver, tout comme Sinon, pourrait avoir menti dans son récit $^{37}$, Desfontaines joue le jeu de l'érudition gratuite et refuse d'interpréter la citation. Il semble également apporter une précision au texte de départ, lorsque Gulliver décrit les «morceaux admirables» que récite son maître houyhnhnm: «[C]'étoit en vérité tantôt le stile d'Homére, tantôt celui de Virgile, tantôt celui de *Milton.» La note indique aux lecteurs que Milton est un «Poëte Anglois, Auteur du Paradise Lost, c'est-à-dire, du Paradis perdu, Poëme

35 Swift, Voyages de Gulliver, trad. Desfontaines, op. cit., 241. Le texte anglais se lit comme suit: «Perjury, Oppression, Subornation, Fraud, Pandarism, and the like Infirmities, were amongst the most excusable Arts they had to mention, and for these I gave, as it was reasonable, great Allowance» (Jonathan Swift, The Travels of Lemuel Gulliver, tome III, 8, 112).

36 Swift, Voyages de Gulliver, trad. Desfontaines, op. cit., 389 («si la Fortune a fait de Sinon un malheureux, son acharnement ne fera pas de lui encore un fourbe et un menteur»; Énéide, trad. Jacques Perret, Paris, Gallimard, 1991, 1977, vol. 1, 41).

37 Voir Allan Bloom, «An Outline of Gulliver's Travels», dans Jonathan Swift, Gulliver's Travels, Robert A. Greenberg, ed., 2e édition, New York et Londres, W. W. Norton \& Company, 1970, 311. 
fameux \& trés-estimé en Angleterre ${ }^{38}$.» Ce dernier passage est absent du texte anglais: il s'agit en fait d'une amplification de Desfontaines, destinée à rendre le texte encore plus anglais (Gulliver ne mentionne jamais Milton).

Même si les ajouts de Desfontaines peuvent servir à accentuer la satire et par conséquent contredire son discours préfaciel et officiel, nous ne nous y attarderons pas. Notre intérêt, nous l'avons dit, ne vise pas ici les «infidélités» de la traduction, mais les notes du texte français. On peut, par contre, se demander quel serait le statut d'une "note du traducteur» qui porte sur un passage qui n'est pas une traduction, mais une pure création du traducteur. Telle la note de l'auteur qui représente un cas limite du paratexte pour Genette, ces notes «traductionnelles fictives", jouant le jeu de la note savante, relèvent de l'auctorial. La mention de Milton comme la référence de la citation latine renforce à la fois le caractère étranger (anglais) du texte et son autorité (mention d'un auteur latin canonique). Ces notes, apparemment référentielles, explicatives et anodines, attirent pourtant l'attention des lecteurs sur les passages en question. Les mentions de Milton ou de Virgile remplissent bien sûr une fonction didactique et érudite (Paradise Lost ne sera traduit qu'en 1729 par Dupré de Saint-Maur). Desfontaines, nourri de lettres classiques comme ses contemporains, aime citer les auteurs latins et, comme tout traducteur, a tendance à expliciter son texte et à montrer qu'il a fait ses «devoirs» de recherche documentaire.

La note sur le "pandarisme» est plus ambiguë, le terme, tant en anglais qu'en français, ne s'entendant plus aussi aisément aujourd'hui. Bay le traduira plus tard par "proxénétisme ${ }^{39}$ ». L'expression renvoie en fait à Pandaros, chef de l'armée troyenne, héros de l'Iliade, dont Chaucer (Troylus and Cryseyde) et Shakespeare (Troilus and Cressida) font un entremetteur. Le terme est trop obscur ou trop familier au XVIII siècle pour être recensé par l'Académie, ce qui permet au traducteur de conserver une allusion qui autrement serait inacceptable ${ }^{40}$.

38 Swift, Voyages de Gulliver, trad. Desfontaines, op. cit., 356.

39 Jonathan Swift, Voyages de Gulliver, trad. André Bay, notes de Jacques Pons, préface de Maurice Pons, Paris, Gallimard et Librairie générale française, coll. «Le livre de poche», 1964, 302.

40 La base de textes littéraires ARTFL (<http://humanities.uchicago.edu/ARTFL $>$ ), dans ses 473 textes pour la période allant de 1700 à 1799 , ne comporte ainsi qu'une seule occurrence du terme, soit chez Piron: «Lé pandar ont biau faire, et n'ête pa no fils; je son toujou leux père» (l'École des pères, dans CEuvres complètes, éd. Rigoley de Juvigny, tome 1, Paris, Lambert, 1776, 151). 
D'autres notes sont plus révélatrices parce qu'elles dépassent le niveau de la simple explicitation linguistique ou culturelle, en ajoutant plus clairement des éléments de la culture d'arrivée. Lorsque Gulliver décrit le caractère "mathématique» des Laputiens, le traducteur ajoute une note ironique. Gulliver rapporte d'abord que

La connoissance quej'avois des mathématiques, m'aida beaucoup à comprendre leurs façons de parler, \& leurs métaphores tirées la plûpart des mathematiques, \& de la Musique; car je suis aussi un peu Musicien*. Toutes leurs idées n'étoient qu'en lignes \& en figures, \& leur galanterie même étoit toute géométrique.

\section{Et Desfontaines renchérit sur la satire:}

*Il ne tiendra pas à moi (dit l'Auteur du traité de la pesanteur, dans une Lettre insérée dans le Merc. de janv. 1727) que tout le monde ne soit géometre, \& que la géometrie ne devienne un stile de conversation, comme la morale, la physique, $l^{\prime}$ histoire et la gazette ${ }^{41}$.

L'auteur de l'article en question s'appliquait à montrer que les corps tombent en décrivant une spirale et non une parabole, et que la géométrie serait comprise de tous, si l'on faisait appel à des exemples clairs. La citation de cet article savant renchérit sur la satire en ajoutant un exemple français de l'engouement pour les mathématiques et la géométrie, ce qui $s^{\prime}$ inscrit d'ailleurs dans une des préoccupations centrales de Desfontaines, puisqu'il dénoncera plus tard avec virulence la vogue de Newton en France ${ }^{42}$.

41 Swift, Voyages de Gulliver, trad. Desfontaines, op. cit., 197. «The Knowledge I had in Mathematics gave me great Assistance in acquiring their Phraseology, which depended much upon that Science and Musick; and in the latter I was not unskilled. Their Ideas are perpetually conversant in Lines and Figures» (Swift, Gulliver's Travels, 1726, op. cit., tome III, ch. 2, 26). Il s'agit de la «Lettre du P.C. à M.B. où il est démontré géometriquement, mais d'une maniere intelligible pour tout le monde, que les Corps jettez d'un lieu en un autre, ne décrivent pas, comme on l'a crû jusqu'ici, une Parabole» (49-58 [signée «C. J.»]).

42 Voir, entre autres sa traduction de The State of Physick (Francis Clifton, 1732, Londres, Bowyer): État de la Médecine ancienne et moderne [...] Par M. Clifton [...], Traduit de l'Anglois par M.L.D.F. (Paris, Quillau, 1742). Non seulement à cause de son opposition à Voltaire, mais aussi à cause de son adhésion au cartésianisme, Desfontaines prendra position contre l'introduction des idées de Newton en France. Nombre de lettres des Observations s'en prennent à Newton en 1738, soit l'année de la Voltairomanie, puis un autre article en 1740: 1738: vol. 15, lettres 213 et 214; 1740: vol. 20, lettre 293. De 
Deux des notes de Desfontaines sont particulièrement longues. Il y explique ses choix traductionnels et semble désamorcer la satire du texte. La première commente le passage où Gulliver raconte à son maître Houyhnhnm comment le commerce appauvrit l'Angleterre et importe les maladies («diseases, folly, and vice $\left.{ }^{43} »\right)$. Le traducteur avoue avoir éprouvé des difficultés linguistiques, mais trouve en même temps des équivalents français aux «scribouillards»:

[C]'était ce qui produisoit les filoux, les voleurs, les M... ., les parjures, les flatteurs, les suborneurs, les faussaires, les faux témoins, les menteurs, les joueurs, les imposteurs, les fanfarons, les mauvais auteurs, les empoisonneurs, les impudiques, les précieux ridicules, les esprits forts ${ }^{44}$.

À ce passage, Desfontaines ajoute une longue note qui semble commencer par un aveu d'impuissance: «*Il est bien surprenant de trouver ici les mauvais Auteurs \& les Précieux ridicules, en si mauvaise compagnie. Mais on n'a pû rendre autrement les mots de Scribling \& de Canting ${ }^{45}$.» $\mathrm{Au}$ risque de se moquer de notre traducteur, il semble étonnant que Desfontaines dise ne pas avoir trouvé d'équivalent pour «canting» et «scribling» (Bay traduira par «scribouillards» et «diffamateurs $\left.{ }^{46} »\right)$, deux pratiques qui, par les démêlés de Desfontaines avec la justice et la nature de ses écrits, ne lui étaient pas étrangères, et ce tant comme responsable que comme victime. En fait, il réduit ici l'énumération des criminels (de vingt et un on passe à dix-sept) en éliminant les doublets (robbing, stealing) et surtout les mentions inacceptables (pimping, whoring).

Mais il y a plus ici. Desfontaines poursuit sa note en soulignant le but de l'auteur et en expliquant la valeur «morale» qu'il trouve dans le passage:

nombreux articles des Jugements attaqueront également Newton (voir Paul Benhamou, Index des Jugements sur les ouvrages nouveaux (1744-1746) de Pierre-François Guyot Desfontaines, Genève, Slatkine, 1986).

43 Swift, Gulliver's Travels, op. cit., IV. 6.

44 Swift, Voyages de Gulliver, trad. Desfontaines, op cit., 326-327. Le passage du texte anglais est le suivant: «Hence it follows of Necessity that vast Numbers of our People are compelled to seek their Livelihood by Begging, Robbing, Stealing, Cheating, Pimping, Forswearing, Flattering, Suborning, Forging, Gaming, Lying, Fawning, Hectoring, Voting, Scribling, Stargazing, Poysoning, Whoring, Canting, Libelling, Free-thinking [...]» (Swift, Gulliver's Travels, op. cit., IV. 6, 83-84).

45 Swift, Voyages de Gulliver, trad. Desfontaines, op. cit., 327.

46 Swift, Voyages de Gulliver, trad. André Bay, op. cit., 374. 
On voit que l'Auteur les a malignement confondus tous ensemble, \& qu'il y a aussi joint exprès les Free-thinking, c'est-à-dire, les esprits forts, ou les incrédules, dont il y a un grand nombre en Angleterre, ainsi qu'en France [nous soulignons]. Au reste, il est aisé de concevoir que le desir de s'avancer dans le monde, produit des esprits libertins, fait faire de mauvais livres, \& porte à écrire d'un style précieux \& affecté, afin de passer pour un bel-esprit ${ }^{47}$.

Desfontaines reprend ici ses propres idées sur la décadence des lettres et surtout sur la préciosité et la néologie. On le voit, le traducteur n'hésite pas à confondre dans l'appareil noticiel les commentaires sur sa traduction, les explications linguistiques ou culturelles, et ses propres réflexions morales et esthétiques sur l'arrivisme et les «mauvais» auteurs, ou sur la néologie ${ }^{48}$. On retrouve ce type de note mixte dans d'autres traductions de Desfontaines, telle celle des Avantures de Joseph Andrews $(1743)^{49}$.

Dans le texte anglais, l'énumération, l'assonance des suffixes en -ing ou l'absence de lien entre les vices et les allusions sexuelles, sont proprement burlesques et en annulent le contenu moral. Desfontaines reproduit bel et bien l'assonance du suffixe -eur, mais, par ses omissions dans la traduction et par son commentaire noticiel, rapproche les propos de Gulliver d'une condamnation morale des «incrédules» et de celle, plus particulière, de l'arrivisme littéraire. Ce passage et toute la note qui l'accompagne sont paradigmatiques du projet de traduction de Desfontaines tel qu'il l'avait défini dans sa préface. On y retrouve tant le projet officiel, soit la transposition de la satire en allégorie morale, que le projet sous-jacent, soit les allusions satiriques inévitables également évoquées entre les lignes dans la préface. Les malfaiteurs universellement condamnables («les voleurs, les pipeurs, les parjures») ne sont pas seuls ici:

47 Swift, Voyages de Gulliver, trad. Desfontaines, op. cit., 327.

48 Le sujet de ce commentaire suffit à l'attribuer à Desfontaines qui avait ridiculisé les précieux et leur écriture dans son Dictionnaire néologique à l'usage des beaux esprits $d u$ siècle. Il reprend ces idées dans une multitude de pamphlets et de plaquettes (anonymes ou non) au cours de sa carrière, pour «censurer» des textes en particulier. Voir jusqu'en 1727: Paradoxes littéraires au sujet de la tragédie d'Inès de Castro (1723); Apologie de m. de Voltaire (1725); Veritez litteraires sur la Tragédie d'Herode et de Mariamne (1725); Lettres d'un rat calotin à Citron Barbet (1727).

49 Les Avantures de Joseph Andrews, et du ministre Abraham Adams [...], par M... Feilding [sic], traduites en François, à Londres, par une Dame Angloise, sur la troisiéme Édition, Londres, A. Millar [Paris?], 1743, 2 vol. 
le traducteur leur ajoute les types humains des «mauvais auteurs» et des "précieux ridicules".

Le deuxième exemple d'un long commentaire poursuit le travail de prévention, le «paratonnerre» contre la critique ${ }^{50}$, si caractéristique des préfaces de l'époque. La note renvoie au passage où Gulliver présente au roi des Houyhnhnms les critères de noblesse des Anglais dans les termes suivants:

J'ajoutai que parmi nous, un corps sec, maigre, décharné, foible, infirme, étoit devenu une marque presque infaillible de noblesse; que même une complexion robuste, \& un air de santé alloient si mal à un homme de qualité, qu'on en concluoit aussi-tôt qu'il étoit le fils de quelque domestique de sa maison, à qui madame sa mère avoit fait part de ses faveurs $[\ldots]^{51}$.

Le traducteur souligne ici, dans une note particulièrement longue, le caractère exagéré de la description, puisqu'il ne faudrait surtout pas prendre à la lettre ce qu'il appelle une "mordante hyperbole», tant en ce qui concerne les Anglais que les Français:

*Je ne crois pas qu'aucun lecteur s'avise de prendre à la lettre cette mordante hyperbole. La noblesse Angloise, selon M. de Saint-Évremond, possede la fine fleur de la politesse, \& on peut dire en général que les seigneurs anglois sont les plus honnêtes gens de l'Europe. Ils ont presque tous l'esprit orné, ils font beaucoup de cas des gens de lettres; ils cultivent les sciences, \& il y en a peu qui ne soient en état de composer des livres. Il ne faut donc prendre cet endroit que comme une pure plaisanterie, ainsi que la plupart des autres traits satyriques répandus dans cet ouvrage. Si quelque esprit plus mal-fait étoit d'humeur de les appliquer serieusement à la noblesse françoise, ce seroit encore une bien plus grande injustice. Ce sont les hommes de néant qui ont fait fortune, ou par leurs pères, ou par eux-mêmes, à qui ces traits peuvent convenir, \& non pas aux

50 Genette, op. cit., 193.

51 Swift, Voyages de Gulliver, trad. Desfontaines, op cit., 333. Le passage du texte anglais est le suivant: «That a weak diseased Body, a meagre Countenance, and sallow Complexion, are no uncommon Marks of a Great Man; and a healthy robust Appearance is so far disgraceful in a Man of Quality, that the World is apt to conclude his real Father to have been one of the Inferiors of the Family [...]. The imperfections of his mind run parallel with those of his body, being a composition of spleen, dullness, ignorance, caprice, sensuality and pride» (Swift, Gulliver's Travels, 1726, op. cit., IV, 6, 97). 
personnes de qualité, qui en France comme ailleurs, sont la portion de l'état, la plus vertueuse, la plus modérée, \& la plus polie ${ }^{52}$.

Le ton est ici le même que dans la préface, mais cette fois-ci le traducteur invoque l'autorité d'un "esprit libre» quelque peu libertin et donc suspect, puisque Saint-Évremond (1613-1703) avait dû quitter la France à cause d'écrits compromettants et vivre en Angleterre de 1675 à sa mort. Mais, si le traducteur prétend que la satire ne saurait s'appliquer à la noblesse, elle peut en revanche viser les parvenus ou les bourgeois, ces «hommes de néant» qui se sont enrichis «ou par leurs pères ou par eux-mêmes».

Doit-on prendre au pied de la lettre ce commentaire d'un Desfontaines lui-même avide d'honneurs et de reconnaissance? Ne faut-il pas plutôt y voir une explicitation ironique destinée à attirer l'attention du lecteur, à souligner que la satire s'applique également à la noblesse française? L'indication qu'il faut lire ce passage "comme une pure plaisanterie, ainsi que la plûpart des autres traits satyriques», fait pencher pour la seconde hypothèse. D'ailleurs, comment la satire pourrait-elle se passer de modèle, de référent? Selon les arguments exposés par Desfontaines dans sa préface, le passage aurait dû être supprimé:

Il est clair que ce Livre n'a point été écrit pour la France, mais pour l'Angleterre, \& que ce qu'il renferme de satyre particulière \& directe ne nous touche point. Après cela, je proteste que si j'eusse trouvé dans mon auteur des traits piquans, dont l'allusion m'eût paru marquée et naturelle, \& dont j'eusse senti le rapport injurieux à quelque personne de ce pays-ci, je les aurois supprimés sans balancer, comme j'ai retranché tout ce qui m'a paru grossier \& indécent ${ }^{53}$.

Desfontaines cherchait en effet dans sa préface à désamorcer toute lecture satirique. Il ne fait allusion à la satire qu'un seule fois et cela pour souligner que le livre ne vise que l'Angleterre. Encore mieux, s'il avait trouvé des traits pouvant s'appliquer aux Français (et on ne peut douter qu'il en ait relevé), il les aurait gommés. Le conditionnel ne trompe pas: pourquoi faire allusion à des traits satiriques qui n'existent pas dans le texte de départ? Si le passage du texte qui fait l'objet de la note avait été «injurieux», il aurait dû être carrément supprimé, et non pas mis en

52 Swift, Voyages de Gulliver, trad. Desfontaines, op cit., 367-368.

53 Swift, Voyages de Gulliver, trad. Desfontaines, op. cit., xxxvi. 
valeur par un commentaire si élaboré. L'allusion aux mœurs des dames de la noblesse couchant avec leurs domestiques est déjà particulièrement indécente pour l'époque, même s'il ne s'agit pas de marquises françaises (et une Mme du Deffand, dédicataire de la traduction, aurait pu en être choquée). Desfontaines souligne pourtant ce passage en lui consacrant une des plus longues notes de sa traduction.

L'allégorie annoncée dans la préface ${ }^{54}$ est ici révisée et devient une satire fondée sur un modèle que le lecteur français connaît bien et qui n'aurait pu être mentionnée de manière aussi explicite au début. Les notes restent prudentes, bien sûr, mais l'insistance sur la noblesse y est transparente. Cette situation s'explique par la fonction même des notes. Alors que la préface est destinée au lectorat potentiel et aux censeurs, les notes, elles, même si leur consultation demeure facultative, relèvent d'une lecture que l'on peut dire actualisée: le lecteur (celui qui a poursuivi la lecture) s'est alors familiarisé avec le sujet et le caractère réel des Voyages, et il sera moins surpris par ce genre de commentaire. Comme le soulignent bien Saïd et Biet, «si la note masque, il arrive aussi qu'elle souligne $^{55}$ ».

Desfontaines met également en valeur un autre passage, qui est d'ailleurs supprimé dans plusieurs éditions anglaises. Il s'agit du passage de la conclusion où Gulliver explique qu'il n'a pas pris possession au nom $\mathrm{du}$ roi des terres qu'il a découvertes et donne comme exemple les conquêtes cruelles du passé (en particulier au Nouveau Monde). Desfontaines précise, cette fois, dans une note bien courte: "Allusion à la conquête du Mexique par les Espagnols, qui exercèrent des cruautés inouies à l'égard des Naturels du païs ${ }^{56}$.» Goulding voit dans l'«absence presque totale d'annotation» une «nouvelle preuve de la hâte et de la négligence» du travail de Desfontaines et s'exclame: «Quelle tentation que l'explication de l'allégorie et des allusions pour un ouvrier consciencieux comme, par exemple, un Van Effen! ${ }^{57}{ }^{\prime}$ Morris reprend le même jugement, quand elle affirme que Desfontaines, incapable de comprendre certaines allusions, «se dispense également de fournir des notes pour expliquer des allégories et des allusions aux événements contemporains,

54 «J'y trouvai des choses amusantes \& judicieuses, une fiction soûtenuë, de fines ironies, des allegories plaisantes, une Morale sensée \& libre, \& par tout une Critique badine \& pleine de sel...» (Swift, Voyages de Gulliver, trad. Desfontaines, op. cit., xiii).

55 Saïd et Biet, loc. cit., 168.

56 Swift, Voyages de Gulliver, trad. Desfontaines, op. cit., 392.

57 Goulding, op. cit., 70 . 
notes souvent essentielles pour la compréhension du texte ${ }^{58}$ ». Le jugement est non seulement anachronique, mais il ne tient nullement compte du projet de traduction défini par Desfontaines lui-même dans sa préface. D'abord, certaines allégories de Swift n'ont simplement pas besoin d'annotation, puisqu'elles restent pour le lecteur de l'époque tout à fait compréhensibles, en français; ce lecteur a ainsi un avantage sur nous qui n'avons plus toujours la capacité de les déchiffrer. Ensuite, et cela est plus important, comme nous l'avons indiqué auparavant, le texte anglais même ne comporte pas de notes. Et, enfin, Desfontaines lui-même avait bel et bien indiqué qu'il avait choisi d'effacer les passages trop satiriques ou les allégories incompréhensibles pour le public français.

Certes, il est possible que notre traducteur partage avec son public l'incapacité à comprendre certaines allusions, ce qui expliquerait l'appareil noticiel restreint. Il ne faut cependant pas oublier que les notes, autoriales traductionnelles ou autres, nuiraient au caractère authentique du récit de voyage homodiégétique. Dans la traduction, ce contrat de véridicité est bien sûr inévitablement rompu par la seule présence du discours du traducteur. Quant au texte de départ, le discours auctorial de Swift en est absent et la préface de Richard Sympson ne sera ajoutée au texte anglais qu'en 1735. Même si le statut du texte change, certaines notes du traducteur peuvent en effet sembler inutiles - sans compter le risque qu'elles poseraient en soulignant trop clairement les passages satiriques. D'abord pour la raison évidente qu'elles ont souvent perdu leur raison d'être lorsque le traducteur supprime ces passages, mais aussi par la nature même du texte que Desfontaines veut donner à son public, soit une allégorie, une fable morale. Goulding et Morris regrettent «le manque d'explicitation de l'allégorie», expression qu'elles emploient toutes les deu ${ }^{59}$. Or, par sa nature même, l'allégorie n'a pas besoin d'être expliquée par l'auteur. La fable peut, quant à elle, offrir clairement une morale, que nous retrouvons ici dans les instances liminaires, en particulier dans la préface qui souligne que Swift, dans les deux premiers voyages, a réussi à «réjouïr \& instruire ses lecteurs, \& [à] leur faire sentir la vanité des grandeurs humaines». Si l'auteur, dans les deux autres voyages, "a eu dessein, encore plus que dans les deux premiers, de censurer plusieurs usages de son pays", selon Desfontaines, «[Il] nous ouvre les yeux sur des vices énormes que nous sommes

58 Morris, loc. cit., 283.

59 Goulding, op. cit., 70; Morris, loc. cit., 283. 
accoutumés à regarder, tout au plus, comme de légers défauts, \& il nous fait sentir le prix d'une raison épurée, \& plus parfaite que la nôtre ${ }^{60}$.»

\section{Conclusion}

Après la dédicace et la longue préface où il critique les Voyages de Gulliver, le silence relatif de Desfontaines comme traducteur dans le texte proprement dit s'explique d'abord par la disparition pure et simple d'allusions tout simplement éliminées dans sa traduction, mais aussi par son refus $d^{\prime}$ insister sur certains passages trop dangereux, et par le simple fait que certaines allusions contemporaines peuvent encore être comprises par les lecteurs français. Desfontaines, premier traducteur de Gulliver, n'a pas pour but, soulignons-le, de produire une édition critique; le rôle de traducteur n'est pas pour Desfontaines dans l'effacement; il modifie le texte de départ, coupe, ajoute, commente, corrige et critique parfois négativement dans les préfaces et les notes, ou même directement dans le texte, et s'en cache assez peu.

Dans les voyages à Laputa et au pays des Houyhnhnms, certaines de ses notes, nous l'avons vu, jouent cependant le jeu de l'édition savante, tandis que d'autres commentent les réflexions de l'auteur. En expliquant certaines références (mention de Milton, allusion à la conquête du Mexique) ou en donnant la référence de citations latines (Énéide), Desfontaines semble se conformer au rôle de traducteur, mais il se joue en fait des règles de la note de traduction, puisque ces explications ne permettent en rien une meilleure compréhension du texte.

Comme bon nombre de traducteurs pragmatiques ou littéraires, Mounin voit dans la note du traducteur «la honte du traducteur ${ }^{61} »$. Dans le cas de la traduction au XVIII ${ }^{\mathrm{e}}$ siècle, la fonction de ces notes est cependant complexe, que ce soit dans la traduction de Swift ou celle de Sophocle. Notre analyse des notes de Desfontaines montre ainsi qu'il existe dans le paratexte des traductions de l'âge classique deux grands types de notes: la note de traduction et la note auctoriale traductionnelle (ou note du «traducteur-commentateur ${ }^{62} »$ ).

60 Swift, Voyages de Gulliver, trad. Desfontaines, op. cit., xxvi-xxvii.

61 Georges Mounin, les Problèmes théoriques de la traduction, Paris, Gallimard, 1963, xi.

62 Georges Kassai, «Pour un dictionnaire des connotations construit sur les notes du traducteur (N.D.T.)", Studi italiani di linguistica teorica e applicata, Pacini, ed., 3, 1994, 509-521, 511. 
La première, classée par Genette parmi les instances allographes, explique normalement un usage exotique ou traduit une citation ou une expression conservée dans sa langue originale (latin, anglais) au sein du texte traduit. Il s'agit essentiellement d'une note qui explique une expression ou une connotation jugée incompréhensible pour le lecteur de la traduction. Ce type de note est parodié par Desfontaines, qui ne fournit pas les renseignements nécessaires en citant Virgile sans traduire ni expliquer le sens de la citation, annote un passage qui est en fait un ajout de sa part, lorsqu'il évoque Milton, ou, enfin, explique qu'il n'a pas traduit "pandarisme».

Le glissement de la note de traduction vers la note auctoriale traductionnelle peut s'effectuer à partir de la note explicative où le traducteur commente ce qui vient d'être lu, comme c'est le cas dans la note sur la noblesse anglaise. Mais, même si une telle note semble avoir pour but de contourner la censure, les commentaires «détonants» de Desfontaines semblent le plus souvent inutiles: une note sur le caractère mathématique des Laputiens renvoie à un article sur la géométrie et une autre mentionne qu'il y a en France autant de «beaux esprits» et d' «esprits forts» qu'en Angleterre. Ces commentaires gratuits renvoient bien sûr à des préoccupations récurrentes dans l'œuvre de Desfontaines, qui dénonce continuellement le «bel esprit» ou la mode des mathématiques, en particulier au moment de la vogue de Newton en France. Mais ces "commentaires» ont également pour fonction de mettre en valeur les éléments satiriques du passage en question.

À l'autre extrême, la note peut prendre la forme d'une explication teintée de désapprobation ou d'ironie qui remet en question les idées de l'auteur. Selon Saïd et Biet, la note qui «se veut objective et scientifique [...] permet le plus souvent le détournement du discours à des fins idéologiques ${ }^{63} »$. La nuance entre la note qui se veut objective et l'explication "teintée de désapprobation ou d'ironie» est à peine nécessaire dans le cas de Desfontaines, dont les notes, qu'elles comportent des commentaires, des explications ou une justification des choix de traduction, ne se veulent pas objectives.

Dans le cas de Desfontaines et des autres traducteurs de son temps, la frontière est donc floue entre la note de traduction et la note de commentaire, ainsi que le montre l'exemple de la note sur la conquête du Mexique qui tient à la fois de l'explication jugée nécessaire par le traducteur et du commentaire par l'auteur français qu'est Desfontaines. 
Les passages les plus dangereux feront alors l'objet d'un commentaire censé désamorcer la satire. La note du traducteur devient ainsi proprement critique et relève de la glose.

Les sujets abordés dans la préface et les notes des Voyages de Gulliver (néologie, «bel esprit», «esprits forts», engouement pour les mathématiques et, bien sûr, l'Angleterre), ainsi que les modifications que Desfontaines apporte au texte, sont liés à une série de prises de position de la part d'un littérateur qui participe à la plupart des querelles de son temps, non seulement par son activité de traducteur, mais aussi comme journaliste, critique littéraire et satiriste. Desfontaines ajoute ainsi un long traité de pédagogie au texte de $\mathrm{Swift}^{64}$ et des commentaires sur la littérature anglaise non seulement dans le Gulliver, mais aussi dans sa traduction de Joseph Andrews et son propre Nouveau Gulliver ${ }^{65}$. Il ridiculise la pédanterie des «néologues» dans la note susmentionnée, comme il l'avait fait dans son Dictionnaire néologique $e^{66}$, et justifie, en apparence, un certain type de roman «moral» et surtout «allégorique» (préface et dédicace du Gulliver, «Lettre de la Dame angloise», en annexe au Joseph Andrews), tout en maintenant dans le texte de ses traductions bon nombre d'allusions plus choquantes. L'étude de ses notes met donc en lumière, non seulement sa conception de la traduction comme activité interventionniste et sa vision d'un lectorat capable de saisir la satire, mais aussi ses prises de position esthétiques et philosophiques, voire idéologiques.

BENOIT LÉGER

Université Concordia

64 Swift, Voyages de Gulliver, trad. Desfontaines, op. cit., II.

65 Desfontaines, le Nouveau Gulliver, Paris, Clouzier et Le Breton, 1730.

66 Desfontaines, Dictionnaire néologique à l'usage des beaux esprits du siècle [...], s.l., 1726. 